\section{A MODIFIED SUCTION DRAINAGE DEVICE}

\section{Dear Editor,}

This refers to the article "A modified suction drainage device" (MJAFI 1995; 51 : 51-2).

I have, on several occasions, used an even simpler device for suction drainage of operation wounds with a high success rate. The suction apparatus is prepared by using an empty glass bottle of any intravenous fluid to which a new intravenous drip set is attached in the usual way. The drip chamber of this set should be without a filter mesh. The other end of the drip set tube is connected to a suction apparatus to create vacuum in the bottle, so that the drip chamber collapses completely. This rough guide ensures adequate vacuum in the bottle. When this is accomplished, the tubing is clamped with a hemostat till it is connected to the drainage tube from the wound. As a drainage tube, one may use a Levine tube or an infant feeding cannula depending upon the requirement in a given case. This tube can be perforated by simply making multiple holes in it. The rear ends of these tubes fit very well into the drip set tube. At the end of the operative procedure the drainage tube is connected to the drip set tube and the clamp is released creating adequate negative suction inside the wound. In the post-operative period, if the drip chamber is found to have regained its original shape, it means the negative pressure inside the bottle is no more sufficient and needs to be re-created in the manner described above.

The main advantages of this system are :

a. One knows whether the negative pressure in the bottle is maintained or not by seeing the drip chamber shape.

b. Use of a drip set obviates the use of glass tubes which are often not readily available in our operation theaters; moreover glass tubes are likely to break.

c. This apparatus is quickly made.

d. It is equally cheap and effective.

However with the increasing popularity plastic IV fluid bottles, the availability of glass bottles is becoming difficult. This will affect the use of this good and cheap indigenons drainage system.

Sqn Ldr PK AGARWAL

Reader in Orthopaedies

AFMC, Pune.

\title{
Author's Reply
}

I read the description of a modified suction drainage system by Sqn Ldr PK Agarwal. This is definitely a better system as maintenance of negative pressure is visible.

The use of an infant feeding tube as a suction catheter may not be adequate due to its small diameter and softness. One can use a $12 \mathrm{G} / 14 \mathrm{G}$ Gibbon catheter or Levine tube and directly attach it to the IV drip set.

\author{
Maj N SRINATH \\ Classified Specialist (Surgery) \\ Military Hospital, Belgaum.
}

